\title{
Die kulturpoetische Funktion des Archivs
}

Es gibt eine erschütternde Episode in Wilhelm Raabes später Erzählung Die Akten des Vogelsangs (1896), wo Velten Andres nach dem Tode seiner Mutter das gesamte Inventar seines Elternhauses verheizt. ${ }^{1}$ Er zerstört damit die letzten Zeugnisse einer vergangenen nachbarschaftlichen Idylle im Vogelsang, einer Gegend, die inzwischen von Fabriken und Vergnügungsetablissements geprägt und zur Unkenntlichkeit modernisiert ist. Veltens Schulfreund Krumhardt, den gutbürgerlichen Ich-Erzähler, ergreift über diesem Autodafé eine ihm selbst unheimliche Begeisterung:

\begin{abstract}
Worin lag nun der Zauber, der mich [...] jeden Tag nach der alten Heimstätte trug, die jetzt zu einer Stätte der Vernichtung geworden war? [...] Wohl selten ist je einem Menschen die Gelegenheit geboten worden, seine „besten Jahre“ in die unruhvolle Gegenwart so zurückzurufen wie mir in Velten Andres' Krematorium. Wie wir im Vogelsang in der Nachbarschaft [...] gelebt hatten, das erfuhr ich nun noch einmal im reichsten Maße und konnte meine Lebensakten in wünschenswertester Weise dadurch vervollständigen. Der Wanderer auf der wankenden Erde [= Velten Andres] schob aus seinem Hausrat kaum ein Stück in den Ofen oder auch auf den Küchenherd, an dem nicht auch für mich eine Erinnerung hing und mit ihm in Flammen aufging und zu Asche wurde. (Raabe 1988, 166)
\end{abstract}

Der Ziegenhainer, die Zerevismütze, das alte Schaukelpferd - alle werden sie noch einmal Anlass, die mit ihnen einst verbundenen Diskurse zu erinnern und aufzuschreiben, ad acta vitae zu legen, die dann jenes Buch ausmachen, das den Titel Die Akten des Vogelsangs trägt. Krumhardt selbst, sein fiktiver Verfasser, ist da übrigens längst vom Vogelsang weggezogen - in der Erzählgegenwart ist er wohnhaft in der Archivstraße!

Mit Archiven hat man es hier in zweierlei unterschiedlicher Form zu tun: zum einen mit dem Elternhaus voller Objekte aus vergangenen Zeiten, zum anderen mit den schriftlichen Akten, in denen ein Zeitzeuge die „Nachbarschaft“, d. h. die Kontiguitätszusammenhänge notiert, in denen diese Objekte einst standen - in jenen Zeiten, da sie noch Bestandteile praktischen Lebens und nicht bloß Sam-

1 Dieser Beitrag ist eine überarbeitete Version meines Aufsatzes Was nicht ins Archiv kommt. Zur Analysierbarkeit kultureller Selektion (vgl. Baßler 2007). Die zugrundeliegende Archivtheorie habe ich ausführlich entwickelt in Die kulturpoetische Funktion und das Archiv (vgl. Baßler 2005). 
melstücke im „Herzensmuseum“ der alten Frau Andres waren. Schon dort konnten sie ja nur deshalb ein Refugium finden, weil jene Zusammenhänge eben im Herzen und im Bewusstsein seiner Bewohnerin gespeichert geblieben waren. Mit deren Ableben aber wären sie derselben erinnerungslosen Zerstörung qua Modernisierung anheimgefallen wie der übrige Vogelsang, wenn sich nicht Archivar Krumhardt eingefunden hätte, um sie in einem anderen Medium, dem des Textes, aufzuzeichnen und diesmal ausdrücklich für die Nachwelt abzuspeichern.

Allerdings wäre dieser Akt der Archivierung wohl niemals erfolgt, wenn Velten Andres nach seiner Heimkehr nicht eben jenes grenzpathologische Zerstörungswerk in Gang gesetzt hätte, das die Bürger des Städtchens zugleich verstört und fasziniert. Es ist Krumhardts braver Ehefrau vorbehalten, den impliziten Horror dessen zu formulieren, was hier vorgeht: „ich habe“, jammert sie,

doch noch letzte Nacht geträumt, auch du habest mich mit unserem Jungen - ich meine unsere letzte Photographie - verbrannt wie er die Bilder seiner Eltern und seiner als ganz kleines Kind verstorbenen Schwester! O bitte, da nimm uns, Ferdi und mich, doch lieber jetzt gleich mit und schieb uns in euren Ofen in deinem Vogelsang! (Raabe 1988, 166)

Ihr Gefühl trügt nicht: Es ist letztlich der Merkwürdigkeit Veltens, eines nach bürgerlichem Maßstab gescheiterten Charakters zwischen Genie und Freak (Lord Byron und Affenmensch), zu verdanken, dass der idyllische Alltag im Vogelsang in Form von Literatur der Nachwelt überliefert wird, während die eigene lebendige Gegenwart der Familie Krumhardt frei von allem Außergewöhnlichen ist und daher - trotz bürgerlicher Routinearchivierung im Medium der Fotografie - aller Voraussicht nach klanglos zum Orkus hinabgehen wird. Raabes leicht marottifizierte Prosa substituiert dabei den lebendigen Zusammenhang von Mutter und Kind durch seine Aufzeichnung und spitzt dadurch bestimmte Charakteristika des Archivierungsprozesses zu bis zur Unerträglichkeit. Wenn man Fotos verbrennt, dann kann man eigentlich auch gleich Menschen verbrennen - so radikal steht es bei Raabe. Und in der Tat: Letztlich teilen Fotos ohne diskursives Umfeld das Schicksal der auf ihnen Abgebildeten. Auch zu vielen der fotografierten Personen aus den Alben, die ich aus meinem Elternhaus aufbewahrt habe, werde ich kaum je mehr die Namen, geschweige denn die Anlässe und Schicksale erfahren. Allenfalls die Textualisierung, die Aufnahme in die Akten, die Verwandlung in Literatur - so legt Raabes Erzählung nahe - vermag diesen Prozess der Isolierung, des Stummwerdens und letztlich der Zerstörung der Dinge des Lebens aufzuhalten. 
When my father died

We put him in the ground

When my father died

It was like a whole library had burned down

heißt es in einem Song von Laurie Anderson (1995). Analoge Vergleiche kann man in letzter Zeit auch immer wieder im Feuilleton lesen, wenn darüber reflektiert wird, dass die letzten Zeugen einer Generation, die Weltkrieg und Holocaust im erwachsenen Alter erleben musste, allmählich aussterben. Was sie nicht mehr zu Protokoll geben, heißt es, ist für die Nachwelt verloren.

Es scheint mir nun kein Zufall, dass das Medium der rettenden Archivierung in solchen Wendungen stets die textuelle Aufzeichnung ist: die Akten, die Bibliothek. Auch wenn die Archivierungsprojekte Spielbergs und die Datenbanken im Netz längst zu akustischen, filmischen und digitalen Aufzeichnungen übergegangen sind - entscheidend sind zwei Eigenschaften: erstens Speicherung (d. h. Objektförmigkeit, Lagerungsfähigkeit und wiederholte Zugänglichkeit) und zweitens Verbalität (d. h. Lesbarkeit). In Kombination ergeben diese beiden Faktoren die Definition eines weiten, aber nicht-metaphorischen Textbegriffes. Für einen so verstandenen Text gilt das Wort Bachtins: „Der Text [...] ist die primäre Gegebenheit [...] allen Denkens in den Humanwissenschaften [...]. Wo kein Text ist, da ist auch nichts, worüber zu forschen oder zu denken wäre“ (Bakhtin 1986, 103). Textualität wird hier also nicht als ein Medium unter anderen, sondern als basale Eigenschaft von Archiven verstanden. Gespeicherte, d. h. einer überprüfbaren wissenschaftlichen Analyse zugängliche Kontexte sind textuell oder sie sind nicht - so ließe sich Bachtins Verdikt archivtheoretisch reformulieren. Um diese kühne, derzeit ein wenig gegen den (performativen, kognitiven, präsenztheoretischen) Trend gesprochene Annahme zu plausibilisieren, sei ein kleiner, aber hoffentlich erhellender Umweg über die Systemtheorie erlaubt.

Auch für Niklas Luhmann erfüllen Texte eine dem Gedächtnis menschlicher ,Bewusstseine‘ analoge Aufgabe: Sie speichern Wissen und halten es transsituativ als Vergleichswissen für je aktuelle Operationen psychischer und sozialer Systeme bereit. Dirk Baecker konkretisiert diesen Gedanken, indem er Kultur überhaupt als dieses Vergleichswissen definiert, mit der Betonung auf „Vergleich“:

„Kultur“ ist das, was unvergleichbare Lebensweisen vergleichbar macht. [...] Der moderne Kulturbegriff ist das Ergebnis der intellektuellen Praxis des Vergleichens. [...] es geht um die scheinbar ganz harmlose intellektuelle Geste, irgend etwas für „interessant“ zu halten und sich mithilfe des Vergleichswissens, das man sich angelesen hat, Gedanken über dieses Interessante zu machen. (Baecker 2000, 47-48) 
Verglichen werden können bedeutsame Kulthandlungen wie das Beten in verschiedenen Religionen, genausogut kann aber auch von Kinderspielzeug, Popmusik oder Essbesteck die Rede sein. Entscheidend ist, dass in Baeckers Modell all dies, selbst die Kulthandlung (das Beten), nicht per se Kultur ist, sondern dass Kultur daraus wird als Ergebnis einer bestimmten Betrachtungsweise. Diese Betrachtungsweise ist der Vergleich. Und wie nichts gleichsam essentiell Kultur ist, so gilt auch umgekehrt, dass es nichts gibt, was per se nicht unter Kultur subsumierbar wäre.

Alles läßt sich vergleichen, alles kann „interessant“ oder „uninteressant“ gemacht werden, von der Frage der Weinbaukunst bis zur Frage der ehelichen Liebe. Alles erscheint doppelt, nämlich einmal als das, was es ist, und einmal als das, was es im Rahmen eines Vergleiches bedeutet. Und natürlich schlagen die Konjunkturen der Bedeutung zurück auf das, was etwas „ist“. Schließlich „ist“ nichts mehr etwas, wenn es nicht zugleich auch etwas „,bedeutet“. (Baecker 2000, 67)

„Alles läßt sich vergleichen“, in einer elaborierten Kultur finden sich keine Dinge, die nicht auch Bedeutung haben, ${ }^{2}$ eine Bedeutung, die ihnen aber wie gesagt nicht ontologisch anhaftet, sondern die ihnen aufgrund einer bestimmten intellektuellen Praxis zugeschrieben werden kann. Wo Baecker über die ethnologische Methode des Kulturvergleichs handelt, benennt er auch, um was für eine Praxis es sich hier handelt:

Eine im strengen Sinne des Wortes ethnologische Kulturbeschreibung dürfte [...] nicht anthropologisch, das heißt mit Referenz auf die Unterschiedlichkeit (und Gleichheit) der beteiligten Menschen, sondern sie müßte semiotisch verfahren, das heißt mit Referenz auf die Zeichen, die den Kulturkontakt so oder anders schwer oder leicht machen. (Baecker 2000, 17-18)

Eine kulturpoetische Betrachtungsweise ethnologisiert gleichsam die eigene Kultur (vgl. Rabinow 1986, 241). Die kulturpoetische Praxis des Vergleichens ist dabei wesentlich eine semiotische, denn „Zeichen“ sind ja eben Dinge im Modus des Vergleichs.

„Kultur ist [demnach] das Ergebnis der intellektuellen Praxis des Vergleichens“ (Baecker 2000, 81). Das Interessante dabei: Textualistisch gefasst ist der

2 Insofern kann es nicht wirklich verwundern, daß Eckhard Henscheid in satirischer Absicht hunderte sogenannter Kulturen zusammentragen konnte (vgl. Henscheid 2001).

3 Baecker setzt sich mehrfach in dieser Weise von der kulturanthropologischen und -soziologischen Tradition in Deutschland ab, etwa von Friedrich Tenbruck und Hans Peter Thurn (vgl. Baecker 2000, 81-82). 
Vergleich, den Baecker im Zentrum seines Kulturbegriffs ansiedelt, zuallererst ein Tropus, und zwar jener Tropus, der Äquivalenzbeziehungen herstellt. Der Vergleich stiftet eine Äquivalenz zwischen den verglichenen Dingen: A ist in gewisser Hinsicht wie (oder nicht wie) B. Äquivalenzbeziehungen aber sind konstitutiv für die paradigmatische Achse jedes Textes, sie definieren genau jene textuelle Dimension, in der die Alternativen zum syntagmatisch notierten Wortlaut gespeichert sind. Die Elemente eines Paradigmas sind dadurch definiert, dass sie einander äquivalent sind - und umgekehrt: Was äquivalent ist, kann ein Paradigma bilden.

Die Differenz interessant/uninteressant, die die Bochumer Schule der Systemtheorie interessanterweise zunächst als Leitdifferenz für das Literatursystem vorgeschlagen hatte, appliziert Baecker auf Kultur allgemein: „,alles“, sagt er, „kann ,interessant‘ oder ,uninteressant' gemacht werden“, indem man es mit anderem vergleicht. Für unsere textuelle Formulierung dieser Theorie muss eine weitere Unterscheidung getroffen werden: Ein syntagmatisch ausgeführter Vergleich mag Dinge interessant oder uninteressant machen, jeder Teil eines Textes bedeutet aber überhaupt nur etwas als Teil eines - genauer gesagt: mindestens eines Paradigmas, also in Bezug auf kulturell verfügbare Vergleichsgrößen. „Alles erscheint doppelt“ - als Ding und als Repräsentation. Sobald man jedoch einmal im interpretativen Modus der Kultur ist, sobald man also kontextualisiert, erscheint es nicht mehr bloß doppelt, sondern vielfach und geradezu „unausschöpfbar“, weil die Paradigmen einer Kultur vielfach und unausschöpfbar sind.

Dem Vergleich im Herzen einer systemtheoretischen Kulturtheorie entspricht also im Herzen einer textualistischen Kulturtheorie das Paradigma. Die Paradigmen einer Kultur sind demnach die vorrätig gehaltenen Aufzeichnungen der „intellektuellen Praxis des Vergleichens“ und bilden zugleich die Folie, vor der jeder neue Text, jede neue Sequenz, jeder neue Vergleich Bedeutung gewinnt. Sie haben den Vorteil, dass sie in Objektform archiviert und daher jederzeit, $d . h$. zeitunabhängig und synchron, ablesbar sind. Dass dieses Archiv dabei materialiter gedacht wird und nicht als latent verfügbarer Code (langue), markiert den Unterschied zwischen einem textualistischen Kulturbegriff und dem einer linguistisch-systemisch bestimmten Sprache.

Dadurch bleibt die Interpretation auch „endlich“, wird also nicht beliebig, denn alles und jedes lässt sich zwar miteinander vergleichen, in einer „konkreten Kultur“ werden (und vor allem: wurden historisch) aber stets nur bestimmte Dinge miteinander verglichen und andere nicht - das eben definiert eine konkrete Kultur und unterscheidet sie von anderen. Was in einer gegebenen Kultur miteinander vergleichbar ist, macht den Sinnhorizont für alle ihre Repräsentationen aus. Dieser kulturelle Sinnhorizont ist also überprüfbarer Analyse zugänglich, jedoch nicht - und darauf kommt es an - als Sinnhorizont einer gegebenen 
Handlung oder Kommunikation, sondern in Gestalt der möglichen Paradigmen zu einem gegebenen Objekt, als Funktion des kulturellen Archivs also.

\section{3}

Der hier propagierte Archivbegriff ist ein denkbar schlichter. Anders als Foucault ist damit nicht irgend ein systemisches, ort- und trägerloses „Gesetz dessen, was gesagt werden kann“ (Foucault 1990, 186-187), kein historisches oder mediales Apriori gemeint, sondern zunächst einmal genau jene „Summe aller Texte, die eine Kultur als Dokumente ihrer eigenen Vergangenheit oder als Zeugnis ihrer beibehaltenen Identität bewahrt hat“ (die Foucault ausdrücklich nicht meint). Mit Boris Groys und gegen Foucault wird das Archiv einer Kultur hier also „als real existierendes verstanden - und in diesem Sinne auch durch die Zerstörung bedroht und deswegen endlich, exklusiv, begrenzt, so daß nicht alle möglichen Aussagen in ihm vorformuliert gefunden werden können“ (Groys 1999, 179).

Dafür aber die wirklichen. Und wenn man statt von Aussagen von Texten spricht und mitbedenkt, dass Texte eine paradigmatische Achse haben, und wenn man diese paradigmatische Achse innerhalb des Korpus, des material gegebenen Archivs selbst ansiedelt als Summe seiner Äquivalenzstrukturen, dann wird die Pointe dieser Entscheidung sichtbar: Die Diskurse und die Texte lassen sich auf ein und demselben Tableau analysieren. Damit und erst damit ist jene von Foucault avisierte Umstellung vollzogen, die ,an die Stelle des Themas der transzendentalen Begründung die Beschreibung der Verhältnisse der Äußerlichkeit“ setzt (Foucault 1990, 182). Das Glück des Positivisten liegt in der Textualität.

Im Archiv sind die Dinge in einer Weise gespeichert, dass man auf sie zugreifen kann, und zwar wiederholt. Im konkreten Falle handelt es sich dabei oft genug um Texte im engeren Sinne, und das nicht zufällig, denn Texte sind ja eben als Instrumente zur Speicherung von Kontiguitätszusammenhängen mit der Möglichkeit des wiederholten Zugriffs entwickelt worden. Andernfalls handelt es sich bei den Dingen im Archiv um Texte genau in dem Maße, wie sie zueinander Paradigmen bilden können. - Dieses Archiv ist die Voraussetzung, die Ausgangsbedingung jeder kulturwissenschaftlichen Arbeit. Was nicht im Archiv ist, kann kulturwissenschaftlich nicht analysiert werden. Im Unterschied $\mathrm{zu}$ anderen Archiv-Begriffen (etwa dem Derridas), die ein Archiv bereits als Ergebnis einer Auswahl, als etwas Zustandegekommenes, als Verwaltungs- und Machtinstrument und darüber hinaus als etwas immer schon Geordnetes, Hierarchisiertes, mit Indices Versehenes beschreiben - was für jedes konkrete Archiv natürlich ebenso zutrifft wie für jeden konkreten Text -, muss eine textualistische Kultur- 
theorie vom Archiv als einer bloßen Sammlung der gegebenen Untersuchungsobjekte ausgehen.

„Wo kein Text ist, da ist auch nichts, worüber $\mathrm{zu}$ forschen oder $\mathrm{zu}$ denken wäre“ (Bakhtin 1986, 103). Aber etwas wird überhaupt erst zum Text oder als Text lesbar durch seine Beziehung zu anderen Texten, intertextuell. „Textualität heißt auch: Praxis des Archivs“ (Ernst 1997, 306), bemerkt Wolfgang Ernst. In kulturpoetischer Lesart heißt das: Das Archiv versammelt die für die Kontextualisierung verfügbaren Texte, es enthält sämtliche Texte, zu denen der Einzeltext in Beziehung gesetzt werden kann, aber noch nicht diese Beziehungen selbst. Das bedeutet, dass es in sich noch nicht indexikalisiert oder strukturiert sein kann. Es ist nicht nur gekennzeichnet durch die „Gleichzeitigkeit seiner Dokumente, die doch aus verschiedenen Zeiten stammen“ (Ernst 2002, 49), sondern ganz generell durch deren strenge Nebenordnung - sans ordre et sans ordre (vgl. Derrida 1997). Es hat, wenn man so will, die Form einer Volltext-Datenbank noch ohne Indices und ohne Links.

Ist es nicht naiv, angesichts einer entwickelten Archiv-Forschung, die von der Handhabung konkreter Archive bis hin zu einer dekonstruktivistischen ArchivTheorie reicht, einen derart schlichten Archiv-Begriff vorzuschlagen? Nun, es sei daran erinnert, was eine kulturwissenschaftlich informierte Literaturwissenschaft leisten soll: Sie soll Texte in ihrer Kultur kontextualisieren. Dazu ist es nötig, in einem ersten Schritt die verfügbaren Dokumente dieser Kultur nebeneinander auf den Tisch zu legen. Das, was dann auf diesem Tisch liegt, nenne ich Archiv. Ohne Zweifel kommen im wirklichen Leben die Texte immer schon irgendwie rubriziert, eingeordnet und bewertet auf uns. Jedes konkrete Archiv ist das Ergebnis entsprechender Prozesse. Aber der erfolgreiche Kunstgriff der New Historicists lag ja zunächst einmal darin, die überkommenen Rubriken, Narrative und Wertungen der Renaissance-Forschung in Frage zu stellen, den Tisch sozusagen wieder frei zu machen für neue Anordnungen. Der vorgeschlagene Archivbegriff ist also gar nicht so abstrakt, wie er zunächst erscheinen mag, wenn man real existierende Archive im Sinn hat. $\mathrm{Zu}$ deren Beschreibung taugt er freilich nicht. Er ist jedoch ausgesprochen konkret im Sinne einer methodologischen Vorgabe: Die Dokumente einer gegebenen Kultur sind zunächst zu kollationieren und nebeneinander anzuordnen. Das entsprechende, Archiv genannte Textkorpus ist Bedingung, Gegenstand und Grenze aller folgenden kulturwissenschaftlichen Operationen. 
Im Zusammenhang eines solchen archivimmanenten Strukturalismus ist das Archiv vor allem die Antwort auf die Frage nach der Analysierbarkeit von kulturellen Äquivalenz- und Kontiguitätsbeziehungen. Das Archiv einer Kultur als Korpus der aus ihr überlieferten Texte beantwortet - wenn Sie so wollen - eine Wo-Frage: Wo sind sie, die Diskurse, die kulturellen Paradigmen, wo ist der semiotische Hintergrund einer Kultur, und zwar materialiter, d. h. sofern sie sich analysieren lassen? Weder Foucaults historisches Apriori mit seinem, wie Groys spottet, ortlosen und immateriellen Träger noch etwa Ecos Konzept einer Enzyklopädie nach dem Quillianschen Modell Q (vgl. Eco 1994, 123-127) beantworten diese Frage nach der Materialität des Paradigmas zufriedenstellend, geschweige denn Luhmanns dynamisches System, das niemals als Struktur analysierbar wird, weil es nicht stillhalten kann. Und Derridas Archiv enthält bereits Propositionen, sozusagen verstandene Sätze, und setzt damit implizit bereits hermeneutische Operationen voraus, deren Bedingung das Archiv m. E. allererst wäre.

Das Archiv, wie es hier entworfen wird, enthält dagegen die Sequenzen einer Kultur ebenso wie deren mögliche Paradigmen. Jeder Einzeltext wird lesbar im Vergleich mit einem Vorrat äquivalenter Möglichkeiten. Analytisch sind diese Möglichkeiten nun aber, wie gesagt, nicht in systemisch-regelhafter Form, sondern allein als Okkurrenzen in positiv vorhandenen Vergleichstexten desselben Archivs zu fassen. Man ahnt, wozu man hier Computer brauchen wird: zur bloßen Quantitäts- und Komplexitätsbewältigung.

Und damit komme ich zur Frage der Analysierbarkeit von Selektion als neben der Kombination wichtigster Operation kultureller Poiesis. In einem strukturalistisch informierten Begriff von Textualität ist Selektion immer schon impliziert. Jakobson nennt bekanntlich die paradigmatische Achse des Textes auch ,Achse der Selektion'. Das ist freilich immer noch produktionsästhetisch und überdies in einem langue/parole-Modell gedacht, noch nicht von einer Materialität des Paradigmas aus, wie sie die Kulturpoetik entwirft. Analytisch gewendet, bezeichnet die Jakobson'sche Selektion denn auch nichts anderes als die Semantisierung von Objekten (Sequenzen, Textstellen) qua Vergleich mit äquivalenten Objekten (Sequenzen, Textstellen). Ein Paradigma ist demnach eine Äquivalenzstruktur im Archiv, d. h. im Korpus der Texte, die man auf vergleichbare Stellen hin durchsucht. Die Sammlung dieser Äquivalenzstellen bezeichnet als eine Art kulturelle Topik die Möglichkeiten dessen, was in einer Kultur anstelle des im manifesten Text Vorgefundenen auch noch sagbar war oder gewesen wäre. Und die Grenzen dieser Operation werden - wie gesagt - definiert durch die materialen Gegebenheiten des Archivs. 
Was von der Foucault'schen Möglichkeitsstruktur übrig bleibt, wenn man sie konkret analysieren möchte, ist also kein historisches Apriori, sondern - ganz positivistisch - eine Sammlung von Vergleichsstellen. Selbstverständlich kann man dann, in einem zweiten Schritt, in einem Abstraktionsvorgang die Eigenschaften dieser Vergleichsstellen als historische Formationsregeln dynamisieren. Aber erstens sehe ich nicht recht den Erkenntniswert einer solchen Operation, und zweitens - und das ist das Entscheidende - ergibt sie, analytisch betrachtet, eben niemals ein Apriori, sondern bleibt gegenüber dem Archiv immer sekundär. Wenn es ein historisches Apriori der Analyse gibt, dann ist das das Archiv.

Nun behauptet etwa Niklas Luhmann, die Offensichtlichkeit überlieferter Texte „verdecke, daß es andere Möglichkeiten gegeben hatte“ (Luhmann 1997, 889). Das ist einerseits richtig. Andererseits aber könnte man einen Text ja gar nicht lesen und schon gar nicht verstehen, wenn man nur seine syntagmatische Achse vor sich hätte. Das bedeutet aber, dass kein Text ohne seine Alternativen bestehen kann; er verdeckt nicht nur nicht, dass es andere Möglichkeiten gegeben hatte, sondern er setzt diese Möglichkeiten zu seinem Verständnis gerade voraus und legt sie damit jeder späteren kommunikativen Anknüpfung potenziell auch wieder mit vor. Andernfalls verlöre er geradezu seine Textualität. Selbst der hochkulturstiftende Gesetzestext, an den Luhmann hier mit Jan Assmann zu denken scheint, etwa das 5. Buch Mose, setzt mit jeder Vorschrift voraus, dass man es auch anders machen kann (aber nicht soll), und mit jeder Aussage, dass es auch anders sein könnte (aber nicht ist), ${ }^{4}$ und Gesellschaften, die die schriftliche Version als verpflichtend aufrechterhalten wollen, müssen zusätzlich Kontrollund Sanktionsmechanismen entwickeln, z. B. eine Inquisition einsetzen, um die reine Lehre $\mathrm{zu}$ bewahren.

Um ein Beispiel zu geben: Meine Begeisterung über die popliterarischen Kataloge von Namen aus Popmusik, Marken- und Medienkultur und anderen Bestandteilen einer Enzyklopädie, die von der deutschen Literatur zuvor allenfalls mit spitzen Fingern angefasst worden war, bezog sich zunächst einmal schlicht darauf, dass diese Dinge überhaupt Eingang in die Literatur fanden. Das Vergleichsarchiv, das Leser von Literatur - auch die professionellen - damit heranzuziehen gezwungen sind, um ihren Texten gerecht zu werden, wurde mit dem Erfolg dieser Popliteratur nach 1995 irreversibel um weite Bereiche unserer globalisierten Gegenwartskultur erweitert. Und zu diesem Vergleichsarchiv gehören dann eben nicht bloß diejenigen Bands und Marken, die in den Texten explizit

4 „Wer darauf verweist, daß für ihn noch die Regel gilt, Whisky nur nach sechs Uhr abends zu trinken, macht damit darauf aufmerksam, daß man damit auch früher schon beginnen könnte“, bemerkt auch Dirk Baecker in seiner Fortführung von Luhmanns Kulturtheorie (Baecker 2000, 24). 
genannt werden, sondern auch jene, zu denen sie im popkulturellen Referenzsystem in Äquivalenz- oder Oppositionsbeziehungen stehen. Und nur weil das so ist, weil einzelne Textstrategien eben ein ganzes Archiv aufzurufen imstande sind, lassen sich in einem zweiten Schritt dann auch die Selektionskriterien benennen und kritisieren, die den jeweiligen Popliteraten und seine Texte kennzeichnen. Damit tut man im Grunde nichts anderes, als diesen Text vor dem von ihm selbst definierten semiotischen Hintergrund $\mathrm{zu}$ semantisieren, also: ihn richtig $\mathrm{zu}$ lesen.

Die Beschreibung von textuellen Selektionsvorgängen ist also, strukturalistisch gefasst, die Definition von Paradigmen. Archivanalytisch gesprochen bedeutet das die Erfassung von Äquivalenzstrukturen im Archiv. Im Vergleich mit den entsprechenden Okkurrenzen in anderen Texten wird die manifeste Textstelle semantisiert. Das ist genau das, was eine kulturwissenschaftliche Literaturwissenschaft tut. Und dabei gilt schlichterdings, wenngleich ernüchternderweise: Was nicht im Archiv ist, kann auch nicht gelesen werden, weder als manifester Text noch als Vergleichstext.

\section{5}

Hier liegt nun ein Einwand nahe, nämlich der, dass insbesondere historische Archive ja niemals vollständig sind, dass viele Dinge einer Kultur - wie der Alltag von Frau Krumhardt und ihren Kindern - niemals aufgezeichnet werden und dass selbst von den Aufzeichnungen und anderen objektförmigen Zeugnissen die allermeisten im Verlaufe der Historie zerstört werden. „Archive, digitale zumal“, behauptet etwa Martin Warnke ganz grundsätzlich, „überdauern nur, wenn sie ständig benutzt werden, wenn eine erhaltende Instanz sie stets neu kodifiziert, interpretiert und bewertet“ (Warnke 2002, 280). Das allerdings scheint mir, über technische Probleme des Erhalts von Datenträgern hinaus, so nicht zutreffend. Ein Archiv ist etwas anderes als ein Gedächtnis. Was im Archiv ist, kann prinzipiell immer auch gelesen werden, selbst wenn es nie dazu gedacht war oder Codes und Lesegeräte erst mühsam rekonstruiert werden müssen. Die ägyptischen Hieroglyphen-Inschriften und Papyri etwa wurden jahrhundertelang weder gelesen noch benutzt. Nur aufgrund ihres reinen Objektcharakters, sozusagen als unsemantisierte Objekte, haben sie überdauert und können heute wieder Teil eines Archivs, also von Vergleichs- und Semantisierungsoperationen sein. Der RosettaStein, ohne den wir vermutlich bis heute diese Schrift nicht lesen könnten, hat als Teil einer Steinmauer überdauert, viele mittelalterliche Texte kennen wir nur, weil sie zufällig auf ein Material geschrieben wurden, das sich später zum Einbinden von Büchern eignete. Archiv und Gedächtnis sind also zu unterscheiden. 
Dennoch bleibt das Faktum, dass niemals die Gesamtheit einer Kultur überliefert wird, und damit die Frage: Wie komme ich zu Hypothesen darüber, was nicht im Archiv ist und warum es nicht im Archiv ist? Es versteht sich ja am Rande, dass Krumhardt seine Erinnerungen an den Vogelsang ebenso wie StuckradBarre seine Mikroenzyklopädien der Popkultur zugleich sammelt und generiert. Sie schaffen die Archivdaten zugleich mit ihrer Vertextung. Sie stellen Kultur her, indem sie sie registrieren. Ebenso klar ist aber etwa seit Nietzsche, dass es aus diesen Daten keinen Weg zurück zu einem Eigentlichen und Ursprünglichen gibt, das hier vertextet worden wäre. Il n'ya pas dehors-texte - es gibt kein Draußen des Archivs; die hermeneutische Figur einer ,Übersetzung aus einem verlorenen Urtext" (Günter Eich) ist von vornherein falsch konzipiert, und wir können auch präzise benennen, warum: weil man nicht mit etwas vergleichen kann, was nicht da ist. Nur im Vergleich mit anderen Sequenzen des Archivs aber lassen sich Lücken und Leerstellen in seinen Texten benennen - oder im Vergleich mit anderen Archiven.

Der Verdacht, Archive seien sozusagen in der Regel Werkzeuge aktiver, machtpolitischer Selektion, scheint mir dagegen so etwas wie ein links-romantischer Topos zu sein. Selbstverständlich gibt es in der Geschichte immer wieder kulturpolitische Ordres, die den Ausschluss und manchmal sogar die Vernichtung bestimmter kultureller Segmente bezwecken. Allein dadurch aber, dass sie, als aktive inquisitorische Maßnahmen, explizit werden müssen, hinterlassen solche Eingriffe jedoch in der Regel Spuren: Befehle, Aktenvermerke, Begründungen, Schwärzungen und andere Palimpseste - denken Sie an jene unheimlichen Auren, die die wegretuschierten Personen auf den Gruppenbildern der Stalin-Ära hinterlassen. Schwarze Listen (also Kataloge) werden angelegt, Giftschränke, ja Museen der zu vernichtenden Kultur. Und dabei ist noch gar nicht von den Dokumenten des Widerstandes die Rede, die solche archivpolitische Unterdrückung provoziert. Aktive Unterdrückung ist zumindest seit der Moderne einer der wichtigsten Diskurs- und Vertextungsanlässe überhaupt. Man könnte geradezu behaupten: Je ausdrücklicher etwas aus dem Archiv einer gegebenen Kultur verdrängt werden soll, desto nachhaltiger wird es sich in dieses Archiv einschreiben - gespenstisch vielleicht, aber darum nicht weniger machtvoll.

Nein, die Verlustquote bei der Archivierung der Geschichte scheint mir in der Regel sehr viel banaleren Ursprungs: Wie in Raabes Vogelsang gehen die Dinge dabei zuerst ihres Gebrauchswertes, ihrer Kontiguitätsrelationen verlustig, und dann auch noch ihres Erinnerungswertes, ihrer paradigmatischen Dimension. Am Ende stehen sie in keinerlei textuellen Zusammenhängen mehr und somit quasi außerhalb der Kultur und werden folgerichtig als Müll aussortiert. Es überlebt nur, was - als Baumaterial oder Buchumschlag - sekundäre Verwendung findet oder was in Erdschichten oder Dachkammern zu liegen kommt, wo es nie- 
manden stört, oder Dinge wie die Bunker des Zweiten Weltkriegs, deren Zerstörung einfach zu teuer ist. Selbst in aestheticis scheint mir, eher als Museumspolitik, der Fall der grottenhässlichen Badezimmerkacheln und Deckenlampen paradigmatisch, die wir beim Einzug in die Altbauwohnung selbstverständlich als Erstes hinauswerfen. Manchmal beschleicht einen dabei kurzzeitig das Gefühl, die Enkel oder Urenkel könnten einen einst dafür verdammen.

Ins Archiv gelangt dagegen, was auch jenseits seines Gebrauchswertes mit Kontiguitäts- und Äquivalenzrelationen versehen bleibt, sprich: was vertextet wird. Dazu eignet sich insbesondere auch die Literatur, die schon Gadamer definiert als „Texte, die nicht verschwinden“, die vielmehr ,im ursprünglichen und eigentlichen Sinne Texte sind“, weil sie nicht im Verstandenwerden sub specie communicationis, sondern erst im wiederholten Zurückkommen auf sie „eigentlich da“ (Gadamer 1984, 46) sind. Womit diese Überlegungen beinahe mit einer Tautologie enden: denn als Text hatten wir ja definiert, was erstens als Objekt (noch) vorhanden und zweitens lesbar ist. Lesbarkeit aber bedeutet Semantisierung im Bezug auf ein Vergleichsarchiv und also - siehe Baecker - die Poiesis von Kultur. Kulturwissenschaftliche Analyse als literaturwissenschaftliche Praxis wäre demnach als Archivanalyse im Modus der Textualität zu konzipieren. Einfacher, meine ich, sind kulturelle Kontexte analytisch nicht zu haben.

In Literatur zur Theorie des Archivs stößt man nicht selten auf kritisch-pessimistische, ja apokalyptische Untertöne. Derrida etwa klagt:

selbst in dem, was die Archivierung ermöglicht und bedingt, werden wir niemals etwas anderes finden als das, was der Destruktion aussetzt und wahrlich mit Destruktion bedroht, indem es a priori das Vergessen und das Archiviolithische in das Herz (coeur) des Monumentes einführt. (Derrida 1997, 26)

Demgegenüber könnte unser pragmatischer Archivbegriff entdramatisierend wirken. Auch er betont zwar die Zerstörbarkeit der Archive, aber damit ist höchst unmetaphorisch die materielle Zerstörbarkeit der Datenträger gemeint. Solange Texte aber in einem intakten Archiv aufbewahrt sind, können sie jederzeit wieder zum materiellen Ausgangspunkt der Analyse werden, und sei es einer Analyse des Vergessens. „Potentielle Aktualität“, sagt Wolfgang Ernst, „ist der Aggregatzustand, in dem die Archivdaten verharren - eine Lage radikaler Latenz“ (Ernst 2002, 122). Sie sind prinzipiell verfügbar, selbst wenn sie lange nicht oder überhaupt noch nie gelesen wurden. Fürchtet euch nicht, könnten sie sagen, wir sind alle noch hier. 


\section{Literaturverzeichnis}

Anderson, Laurie. The Ugly One with the Jewels and Other Stories. Warner Brothers, 1995.

Baecker, Dirk. Wozu Kultur? Berlin: Kulturverlag Kadmos, 2000.

Bakhtin, Mikhail M. „The Problem of the Text in Linguistics, Philology, and the Human Sciences. An Experiment in Philosophical Analysis“. Speech Genres and Other Late Essays. Hg. Caryl Emerson und Michael Holquist. Austin, Texas: Univ. of Texas Press, 1986. 103-131.

Baßler, Moritz. Die kulturpoetische Funktion und das Archiv. Eine literaturwissenschaftliche Text-Kontext-Theorie. Tübingen: Francke, 2005.

Baßler, Moritz. „Was nicht ins Archiv kommt. Zur Analysierbarkeit kultureller Selektion“. Die Szene der Gewalt. Bilder, Codes und Materialitäten. Hg. Daniel Tyradellis und Burkhardt Wolf. Frankfurt a. M. [u .a.]: P. Lang, 2007. 61-75.

Derrida, Jacques. Dem Archiv verschrieben. Eine Freudsche Impression. Übers. Hans-Dieter Gondek und Hans Naumann. Berlin: Brinkman + Bose, 1997.

Eco, Umberto. Einführung in die Semiotik. Übers. Jürgen Trabant. 8. Aufl. München: Fink, 1994. Ernst, Wolfgang. „,Nothing but Text‘? Wissensarchäologische Anmerkungen zum Verhältnis von Kultursemiotik, New Historicism und Archiv“. Poststrukturalismus. Herausforderung an die Literaturwissenschaft. Hg. Gerhard Neumann. Stuttgart, Weimar: Metzler, 1997. 290-306.

Ernst, Wolfgang. Das Rumoren der Archive. Ordnung aus Unordnung. Berlin: Merve, 2002.

Foucault, Michel. Archäologie des Wissens. Übers. Ulrich Köppen. 4. Aufl. Frankfurt a. M.: Suhrkamp, 1990.

Gadamer, Hans-Georg. „Text und Interpretation“. Text und Interpretation. Deutsch-französische Debatte. Hg. Philippe Forget. München: Fink, 1984. 24-55.

Groys, Boris. Über das Neue. Versuch einer Kulturökonomie. Frankfurt a. M.: Fischer Taschenbuch Verl., 1999.

Henscheid, Eckhard. Alle 756 Kulturen. Eine Bilanz. Frankfurt a. M.: Zweitausendeins, 2001. Luhmann, Niklas. Die Gesellschaft der Gesellschaft. 2 Bde. Frankfurt a. M.: Suhrkamp, 1997. Raabe, Wilhelm. Die Akten des Vogelsangs. Erzählung. Stuttgart: Reclam, 1988.

Rabinow, Paul. „Representations Are Social Facts. Modernity and Post-Modernity in Anthropology “. Writing Culture. The Poetics and Politics of Ethnography. Hg. James Clifford und George E. Marcus. Berkeley, Los Angeles, London: Univ. of California Press, 1986. 235-261.

Warnke, Martin. „Digitale Archive“. Archivprozesse. Die Kommunikation der Aufbewahrung. Hg. Hedwig Pompe und Leander Scholz. Köln: DuMont, 2002. 269-281. 
